\title{
Taxa de Cruzamento de Nível e Duração Média de Desvanecimento para Canais $\alpha-\mu$ usando Técnicas de Combinação por Diversidade
}

\author{
Daniel Benevides da Costa, Student Member, IEEE, Michel Daoud Yacoub e José Cândido Silveira Santos Filho
}

\begin{abstract}
Resumo-Este artigo apresenta expressões para a taxa de cruzamento de nível e duração média de desvanecimento de combinadores multirramos por seleção pura, ganho igual e razão máxima operando em canais de desvanecimento $\alpha-\mu$ (também chamados Gama generalizados) independentes e não-idênticos. As expressões são obtidas em forma fechada, para combinadores por seleção pura, e na forma integral, para combinadores por ganho igual e razão máxima. Para estes dois últimos cenários de combinação, aproximações precisas e em forma fechada são propostas. Os resultados analíticos são validados reduzindo as expressões gerais para casos particulares conhecidos e por meio de simulação.
\end{abstract}

Palavras-Chave-Métodos de aproximação, duração média de desvanecimento, técnicas de diversidade, canais de desvanecimento generalizados, taxa de cruzamento de nível.

Abstract-This paper derives exact expressions for the level crossing rate and average fade duration of multibranch pureselection, equal-gain, and maximal-ratio combiners operating over independent non-identical $\alpha-\mu$ (also called generalized Gamma or Stacy) fading channels. The derived expressions are in closed form for pure-selection combining and in integral form for equal-gain and maximal-ratio combining. For the two latter schemes, accurate closed-form approximations are then provided. The analytical results are validated by reducing the general expressions to known particular cases and, more generally, by means of simulation.

Keywords-Approximation methods, average fade duration, diversity methods, generalized fading channels, level crossing rate.

\section{INTRODUÇÃO}

O modelo de desvanecimento $\alpha-\mu$ foi recentemente proposto em [1], [2] levando em consideração dois importantes fenômenos inerentes à rádiopropagação, que são a nãolinearidade e a influência do número de clusters. Além de sua flexibilidade e simplicidade matemática, o modelo provê um bom ajuste a medidas de campo, como demonstrado em inúmeras campanhas de medição. A distribuição que rege este modelo, distribuição $\alpha$ - $\mu$, é escrita em termos de parâmetros físicos, $\alpha$ e $\mu$, que descrevem a não-linearidade do meio de propagação e o número de clusters de ondas de multipercurso, respectivamente. Esta distribuição é também como Gama generalizada ou Stacy [1], [2].

Daniel Benevides da Costa, Michel Daoud Yacoub e José Cândido Silveira Santos Filho, Departamento de Comunicações, Faculdade de Engenharia Elétrica e Computação, Universidade Estadual de Campinas, Campinas, Brasil, E-mails: daniel@wisstek.org, michel@wisstek.org, candido@wisstek.org. Este trabalho foi parcialmente financiado pela FAPESP (05/59259-7).
Em sistemas sem fio, a envoltória do sinal recebido varia aleatoriamente devido ao desvanecimento por multipercurso, e técnicas de diversidade são geralmente utilizadas para combater esta variação aleatória do nível de sinal. A taxa de cruzamento de nível (LCR) e a duração média de desvanecimento (AFD) são métricas importantes que avaliam o desempenho dinâmico de sistemas de diversidade. Por esta razão, tais métricas têm sido amplamente investigadas na literatura para os canais de desvanecimento mais conhecidos [3], [4], [5], [6]. Um dos propósitos deste artigo é obter expressões exatas para a LCR e AFD de combinadores multirramos por ganho igual e por razão máxima operando em canais de desvanecimento $\alpha-\mu$ independentes e não-idênticos. Devido à dificuldade inerente da formulação exata, no qual a LCR e AFD são expressas em termos de múltiplas integrais, aproximações precisas e em forma fechada são propostas para tais métricas. Como será visto através de resultados numéricos, as diferenças entre as curvas exatas e aproximadas são imperceptíveis. Além disso, baseando em alguns resultados da literatura, expressões simples e em forma fechada para a LCR e AFD usando a técnica combinação por seleção pura (PSC) são obtidas. Os resultados analíticos apresentados são validados reduzindo as expressões gerais para alguns casos particulares, no qual as soluções são conhecidas, e por meio de simulação.

Este artigo está estruturado da seguinte forma. A Seção II apresenta o modelo de desvanecimento para canais $\alpha-\mu$ e introduz algumas estatísticas importantes utilizadas ao longo do artigo. Na Seção III são obtidas expressões para a LCR e AFD de canais de desvanecimento $\alpha-\mu$ usando as técnicas PSC, combinação por ganho igual (EGC) e combinação por razão máxima (MRC). Como para as técnicas EGC e MRC as soluções exatas são dadas em termos de múltiplas integrais, na Seção IV são propostas aproximações simples e em forma fechada para a LCR e AFD de tais métricas. Na Seção V são plotados alguns resultados numéricos e finalmente na Seção VI concluímos o artigo. Um Apêndice no final do artigo mostra a dedução da variância da função densidade de probabilidade (PDF) condicional das envoltórias na entrada do combinador.

\section{O Modelo De Desvanecimento $\alpha-\mu$}

Nesta seção, o modelo de desvanecimento $\alpha$ - $\mu$ proposto em [1], [2] será revisto. A condição de propagação $\alpha-\mu$ considera um sinal composto por clusters de ondas de multipercurso em um ambiente não-homogêneo. Dentro de cada um dos clusters, as fases das ondas espalhadas são aleatórias, com o espalhamento dos atrasos dos diversos clusters sendo relativamente 
grande. Além disso, os clusters de ondas de multipercurso possuem ondas espalhadas com potências idênticas. A envoltória resultante é obtida como uma função não-linear do módulo da soma das componentes de multipercurso. Tal não-linearidade é manifestada em termos de um parâmetro de potência, tal que a intensidade do sinal resultante é obtida não apenas como o módulo da soma das componentes de multipercurso, mas como este módulo elevado a um certo expoente.

Assumindo que o sinal recebido no $i$-ésimo ramo $(i=$ $1, \ldots, M)$ inclui um certo número $n_{i}$ de clusters de multipercurso, a envoltória $\alpha-\mu$ resultante $R_{i}$ no $i$-ésimo ramo é escrita como [1], [2]

$$
R_{i}^{\alpha_{i}}=\sum_{l=1}^{n_{i}}\left(X_{i l}^{2}+Y_{i l}^{2}\right),
$$

onde $\alpha_{i}>0$ é o parâmetro de potência, $X_{i l}$ e $Y_{i l}$ são processos gaussianos mutuamente independentes de médias nula com variâncias idênticas $V\left(X_{i l}\right)=V\left(Y_{i l}\right)=\sigma_{i}^{2}$, onde $V(\cdot)$ denota o operador variância. A PDF de $R_{i}$ é dada por [1], [2]

$$
f_{R_{i}}\left(r_{i}\right)=\frac{\alpha_{i} \mu_{i}^{\mu_{i}} r_{i}^{\alpha_{i} \mu_{i}-1}}{{\hat{r_{i}}}^{\alpha_{i} \mu_{i}} \Gamma\left(\mu_{i}\right)} \exp \left(-\mu_{i} \frac{r_{i}^{\alpha_{i}}}{{\hat{r_{i}}}^{\alpha_{i}}}\right) \text {, }
$$

onde $\hat{r}_{i}=\sqrt[\alpha_{i}]{E\left(R_{i}^{\alpha_{i}}\right)}=\sqrt[\alpha_{i}]{2 \mu_{i} \sigma_{i}^{2}}$ é o valor médio da raiz $\alpha_{i}$-ésima de $R_{i}^{\alpha_{i}}, E(\cdot)$ denota o operador esperança, $\Gamma(z)=\int_{0}^{\infty} t^{z-1} \exp (-t) d t$ é a função Gama, e $\mu_{i}>0$ é a extensão real do parâmetro $n_{i}$, dado pelo inverso da variância normalizada de $R_{i}^{\alpha_{i}}$, isto é,

$$
\mu_{i}=\frac{E^{2}\left(R_{i}^{\alpha_{i}}\right)}{V\left(R_{i}^{\alpha_{i}}\right)} .
$$

Para $\mu_{i}=1$, (2) reduz a PDF de Weibull, enquanto para $\alpha_{i}=2$ reduz-se a PDF de Nakagami- $m$. A partir de (2), o $k$-ésimo momento $E\left(R_{i}^{k}\right)$ pode ser expresso como

$$
E\left(R_{i}^{k}\right)=\hat{r}_{i}^{k} \frac{\Gamma\left(\mu_{i}+k / \alpha_{i}\right)}{\mu_{i}^{k / \alpha_{i}} \Gamma\left(\mu_{i}\right)} .
$$

A função de distribuição cumulativa (CDF) $F_{R_{i}}(\cdot)$ de $R_{i}$ é dada por [1], [2]

$$
F_{R_{i}}\left(r_{i}\right)=\frac{\Gamma\left(\mu_{i}, \mu_{i} r_{i}^{\alpha_{i}} / \hat{r}_{i}^{\alpha_{i}}\right)}{\Gamma\left(\mu_{i}\right)},
$$

onde $\Gamma(z, y)=\int_{0}^{y} t^{z-1} \exp (-t) d t$ é a função Gama incompleta.

Para espalhamento isotrópico, as derivadas temporais $\dot{X}_{i l}$ e $\dot{Y}_{i l}$ de $X_{i l}$ e $Y_{i l}$, respectivamente, são variáveis gaussianas de médias nula e variâncias $\dot{\sigma}_{i}{ }^{2}=2 \pi^{2} f_{m}^{2} \sigma_{i}^{2}$ [7], onde $f_{m}$ é o desvio Doppler máximo em Hz. Conseqüentemente, baseado em (1), a PDF condicional $f_{\dot{R}_{i} \mid R_{i}}(\cdot \mid \cdot)$ da derivada temporal de $R_{i}$, expressa por $\dot{R}_{i}$, dado $R_{i}$, é escrita como

$$
f_{\dot{R}_{i} \mid R_{i}}\left(\dot{r}_{i} \mid r_{i}\right)=\frac{1}{\sqrt{2 \pi} \sigma_{\dot{R}_{i}}} \exp \left(-\frac{1}{2}\left(\frac{\dot{r_{i}}}{\sigma_{\dot{R_{i}}}}\right)^{2}\right),
$$

no qual $\sigma_{\dot{R}_{i}}^{2}=\frac{r_{i}^{2-\alpha_{i}}}{\alpha_{i}^{2} \mu_{i}} \Omega_{i} 4 \pi^{2} f_{m}^{2}$ (ver Apêndice I) e $\Omega_{i}=\hat{r}_{i}{ }^{\alpha_{i}}$. Tais resultados foram obtidos e usados em [2] para calcular a
LCR $N_{R_{i}}(r)$ e AFD $T_{R_{i}}(r)$ da envoltória $\alpha-\mu R_{i}$, ou seja,

$$
\begin{aligned}
& N_{R_{i}}(r)=\frac{\sqrt{2 \pi} f_{m} r^{\alpha_{i}\left(\mu_{i}-1 / 2\right)} \mu_{i}^{\mu_{i}-1 / 2}}{\Gamma\left(\mu_{i}\right) \Omega_{i}^{\mu_{i}-1 / 2}} \exp \left(-\frac{\mu_{i} r^{\alpha_{i}}}{\Omega_{i}}\right), \\
& T_{R_{i}}(r)=\frac{\Gamma\left(\mu_{i}, \mu_{i} r^{\alpha_{i}} / \Omega_{i}\right) \Omega_{i}^{\mu_{i}-1 / 2}}{\sqrt{2 \pi} f_{m} r^{\alpha_{i}\left(\mu_{i}-1 / 2\right)} \mu_{i}^{\mu_{i}-1 / 2}} \exp \left(\frac{\mu_{i} r^{\alpha_{i}}}{\Omega_{i}}\right) .
\end{aligned}
$$

\section{TAXa de Cruzamento de Nível E DuRAÇÃo MÉdia de DesvaneCimento}

A LCR é definida como o número médio de cruzamentos do sinal por segundo em um dado nível, na direção positiva ou negativa. Sendo $\dot{R}$ a derivada temporal da envoltória $R$ e $r$ o nível de cruzamento, a LCR é estimada como [6]

$$
N_{R}(r)=\int_{0}^{\infty} \dot{r} f_{R, \dot{R}}(r, \dot{r}) d \dot{r}
$$

onde $f_{R, \dot{R}}(\cdot, \cdot)$ é a PDF conjunta de $R$ e $\dot{R}$. A AFD é definida como o tempo médio que a envoltória recebida permanece abaixo de um dado limiar $r$ após cruzá-lo na direção negativa, sendo expresso por

$$
T_{R}(r)=\frac{F_{R}(r)}{N_{R}(r)}
$$

onde $F_{R}(\cdot)$ é a CDF de $R$. Nas seções seguintes, $R$ e $\dot{R}$ representam a envoltória na saída do combinador e sua derivada temporal, respectivamente.

\section{A. Combinação por Seleção Pura}

Em PSC, os sinais recebidos são continuamente monitorados tal que o melhor sinal é selecionado. Dessa forma, a envoltória na saída do combinador $R$ pode ser escrita como

$$
R=\max _{i=1, \ldots, M}\left\{R_{i}\right\}
$$

Em [8], uma formulação geral para sistemas PSC operando com canais de desvanecimento independentes foi apresentada, como descrita abaixo

$$
N_{R}(r)=\sum_{i=1}^{M} N_{R_{i}}(r) \prod_{\substack{j=1 \\ j \neq i}}^{M} F_{R_{j}}(r)
$$

Neste caso, $F_{R_{j}}(\cdot)$ e $N_{R_{i}}(\cdot)$ são dados por (5) e (7), respectivamente. Da mesma forma, desde que para ramos independentes a seguinte relação $F_{R}(r)=\prod_{i=1}^{M} F_{R_{i}}(r)$ é válida, substituindo isso e (12) em (10), e após manipulações algébricas, pode ser mostrado que

$$
T_{R}^{-1}(r)=\sum_{i=1}^{M} T_{R_{i}}^{-1}(r)
$$

onde $T_{R_{i}}(r)$ é dado por (8). Vale a pena ressaltar que (13) é de fato geral e se aplica a qualquer cenário de desvanecimento com ramos independentes. 
1) Combinação por Ganho Igual: Em EGC, os sinais recebidos com envoltórias $R_{i}$ são cofasados e adicionados tal que a envoltória na saída do combinador $R$, já levando em conta a potência do ruído resultante na saída do combinador, é escrita como

$$
R=\frac{1}{\sqrt{M}} \sum_{i=1}^{M} R_{i}
$$

Diferenciando ambos os lados de (14), temos

$$
\dot{R}=\frac{1}{\sqrt{M}} \sum_{i=1}^{M} \dot{R}_{i}
$$

Em [5], [10], foi mostrado que $f_{R, \dot{R}}(\cdot, \cdot)$ pode ser escrito em termos da PDF conjunta de $R_{1}, \ldots, R_{M}$ e $\dot{R}$ como

$$
\begin{aligned}
& f_{R, \dot{R}}(r, \dot{r})=\sqrt{M} \overbrace{\int_{0}^{\sqrt{M} r} \int_{0}^{\sqrt{M} r-r_{M}} \ldots \int_{0}^{\sqrt{M} r-\sum_{i=3}^{M} r_{i}}}^{M-1} \\
& f_{R_{1}, R_{2}, \ldots, R_{M}, \dot{R}}\left(\left(\sqrt{M} r-\sum_{i=2}^{M} r_{i}\right), r_{2}, \ldots, r_{M}, \dot{r}\right) d r_{2} \ldots d r_{M} .
\end{aligned}
$$

Usando propriedades da teoria de probabilidade [9], temos que

$$
\begin{aligned}
& f_{R_{1}, R_{2}, \ldots, R_{M}, \dot{R}}\left(r_{1}, r_{2}, \ldots, r_{M}, \dot{r}\right)= \\
& f_{\dot{R} \mid R_{1}, R_{2}, \ldots, R_{M}}\left(\dot{r} \mid r_{1}, r_{2}, \ldots, r_{M}\right) \times f_{R_{1}, \ldots, R_{M}}\left(r_{1}, \ldots, r_{M}\right) .
\end{aligned}
$$

Devido os ramos serem assumidos independentes, então a PDF conjunta das envoltórias de entrada pode ser escrita como o produto das PDFs marginais, ou seja,

$$
f_{R_{1}, \ldots, R_{M}}\left(r_{1}, \ldots, r_{M}\right)=\prod_{i=1}^{M} f_{R_{i}}\left(r_{i}\right),
$$

onde cada $f_{R_{i}}(\cdot)$ é dado por (2). Por outro lado, a partir de (15), perceba que

$$
f_{\dot{R} \mid R_{1}, R_{2}, \ldots, R_{M}},\left(\dot{r} \mid r_{1}, r_{2}, \ldots, r_{M}\right) \sim N\left(0, \sum_{i=1}^{M} \sigma_{\dot{R}_{i}}^{2} / M\right),
$$

com $\sigma_{\dot{R}_{i}}^{2}=\frac{r_{i}^{2-\alpha_{i}}}{\alpha_{i}^{2} \mu_{i}} \Omega_{i} 4 \pi^{2} f_{m}^{2}$ (ver Apêndice I) e onde $N(a, b)$ representa uma distribuição gaussiana de média $a$ e variância b. Agora, com (17), (18) e (19) em (16), e então substituindo isto em (9), segue, após manipulações algébricas, que

$$
\begin{aligned}
& N_{R}(r)=\sqrt{2 \pi} f_{m} \overbrace{\int_{0}^{\sqrt{M} r} \int_{0}^{\sqrt{M} r-r_{M}} \ldots \int_{0}^{\sqrt{M} r-\sum_{i=3}^{M} r_{i}}}^{M-1} \\
& \sqrt{\frac{\left(\sqrt{M} r-\sum_{i=2}^{M} r_{i}\right)^{2-\alpha_{1}} \Omega_{1}}{\alpha_{1}^{2} \mu_{1}}+\sum_{i=2}^{M} \frac{r_{i}^{2-\alpha_{i}} \Omega_{i}}{\alpha_{i}^{2} \mu_{i}}} \\
& \times f_{R_{1}}\left(\sqrt{M} r-\sum_{i=2}^{M} r_{i}\right) \prod_{i=2}^{M} f_{R_{i}}\left(r_{i}\right) d r_{2} \ldots d r_{M-1} d r_{M} .
\end{aligned}
$$

Para calcular a AFD, falta encontrarmos a CDF $F_{R}(\cdot)$ de $R$. Isso pode ser feito integrando a PDF conjunta
$f_{R_{1}, \ldots, R_{M}}(\cdot, \ldots, \cdot)$ de $R_{i}$ sobre o volume $M$-dimensional limitado pelo hiperplano $\sqrt{M} r=\sum_{i=1}^{M} r_{i}$ e suas coordenadas. Usando um procedimento similar ao aplicado em [5], [10], segue que

$$
F_{R}(r)=\int_{0}^{\sqrt{M} r} \int_{0}^{\sqrt{M} r-r_{M}} \ldots \int_{0}^{\sqrt{M} r-\sum_{i=3}^{M} r_{i}} \int_{0}^{\sqrt{M} r-\sum_{i=2}^{M} r_{i}}
$$

Substituindo (20) e (21) em (10), a AFD é obtida.

\section{B. Combinação por Razão Máxima}

Em MRC, os sinais recebidos são cofasados, cada sinal é amplificado apropriadamente por combinação ótima e os sinais resultantes são então adicionados tal que a envoltória $R$, na saída do combinador, é dada por

$$
R=\sqrt{\sum_{i=1}^{M} R_{i}^{2}}
$$

A derivada temporal $\dot{R}$ de $R$ pode ser expressa como

$$
\dot{R}=\sum_{i=1}^{M} \frac{R_{i}}{R} \dot{R}_{i}
$$

A análise para MRC segue o mesmo raciocínio à realizada para EGC, porém o hiperplano usado para o cálculo das estatísticas é $r^{2}=\sum_{i=1}^{M} r_{i}^{2}$. Segue então que

$f_{R, \dot{R}}(r, \dot{r})=\overbrace{\int_{0}^{r} \int_{0}^{\sqrt{r^{2}-r_{M}^{2}}} \ldots \int_{0}^{\sqrt{r^{2}-\sum_{i=3}^{M} r_{i}^{2}}}}^{\frac{r}{\sqrt{r^{2}-\sum_{i=2}^{M} r_{i}^{2}}}}$
$\times f_{R_{1}, R_{2}, \ldots, R_{M}, \dot{R}}\left(\left(\sqrt{r^{2}-\sum_{i=2}^{M} r_{i}^{2}}\right), r_{2}, \ldots, r_{M}, \dot{r}\right) d r_{2} \ldots d r_{M}$,

$$
\begin{aligned}
F_{R}(r)=\int_{0}^{r} \int_{0}^{\sqrt{r^{2}-r_{M}^{2}}} & \ldots \int_{0}^{\sqrt{r^{2}-\sum_{i=3}^{M} r_{i}^{2}}} \int_{0}^{\sqrt{r^{2}-\sum_{i=2}^{M} r_{i}^{2}}} \\
& \prod_{i=1}^{M} f_{R_{i}}\left(r_{i}\right) d r_{1} d r_{2} \ldots d r_{M-1} d r_{M},
\end{aligned}
$$

e, a partir de (23)

$$
f_{\dot{R} \mid R_{1}, R_{2}, \ldots, R_{M}},\left(\dot{r} \mid r_{1}, r_{2}, \ldots, r_{M}\right) \sim N\left(0, \sum_{i=1}^{M} r_{i}^{2} \sigma_{\dot{R}_{i}}^{2} / r^{2}\right)
$$


tal que, usando (17) e (18), temos

$$
\begin{aligned}
& N_{R}(r)=\sqrt{2 \pi} f_{m} \overbrace{\int_{0}^{r} \int_{0}^{\sqrt{r^{2}-r_{M}^{2}}} \ldots \int_{0}^{\sqrt{r^{2}-\sum_{i=3}^{M} r_{i}^{2}}}}^{M-1} \\
& \frac{1}{\sqrt{r^{2}-\sum_{i=2}^{M} r_{i}^{2}}} \sqrt{\frac{\left(r^{2}-\sum_{i=2}^{M} r_{i}^{2}\right)^{\frac{4-\alpha_{1}}{2}} \Omega_{1}}{\alpha_{1}^{2} \mu_{1}}+\sum_{i=2}^{M} \frac{r_{i}^{4-\alpha_{i}} \Omega_{i}}{\alpha_{i}^{2} \mu_{i}}} \\
& \times f_{R_{1}}\left(\sqrt{\left.r^{2}-\sum_{i=2}^{M} r_{i}^{2}\right) \prod_{i=2}^{M} f_{R_{i}}\left(r_{i}\right) d r_{2} \ldots d r_{M-1} d r_{M} .}\right.
\end{aligned}
$$

Substituindo (27) e (25) em (10), a AFD é obtida.

\section{APROXIMAÇÕES EM Forma FECHADA}

As formulações desenvolvidas nas seções anteriores são gerais e exatas. Por outro lado, exceto para aqueles casos especiais para os quais expressões em forma fechada são encontradas (não mostradas aqui devido a limitação de espaço), soluções dadas em função de múltiplas integrais podem não ser tão interessantes do ponto de vista computacional. Sendo assim, é certamente desejável encontrar aproximações precisas que possam ser utilizadas para substituir tais integrais. Em [11], foi mostrado que a soma de variáveis Weibull pode ser aproximada por uma variável $\alpha-\mu$. Aqui, com o intuito de obter aproximações simples e em forma fechada para a LCR e AFD de sistemas EGC e MRC em canais de desvanecimento $\alpha-\mu$, nós estendemos a idéia apresentada em [11] aproximando a soma de processos aleatórios $\alpha-\mu$ por um outro processo aleatório $\alpha-\mu$. A motivação para isso vem do fato de que a soma de potências $\alpha-\mu$ segue também uma distribuição $\alpha$ $\mu$. Sendo mais claro, assumindo que $Z_{i}, i=1, \ldots, M$ são variáveis $\alpha-\mu$ i.i.d. (i.i.d., do inglês independent identically distributed) com parâmetros $\alpha, \mu$ e $\hat{r}$, então, a partir do modelo proposto, $Z^{\alpha}=\sum_{i=1}^{M} Z_{i}^{\alpha}$ é também distribuído $\alpha-\mu$ com parâmetros $\alpha, \mu M$ e $\sqrt[\alpha]{\hat{r}^{\alpha} M}$.

Nossa proposta é então aproximar a LCR e a AFD exatas de sistemas EGC e MRC obtidas na seção anterior pela LCR e AFD de uma dada variável $\alpha-\mu$, isto é [1], [2]

$$
\begin{aligned}
N_{R}(r) & \approx \frac{\sqrt{2 \pi} f_{m} r^{\alpha(\mu-1 / 2)} \mu^{\mu-1 / 2}}{\Gamma(\mu) \Omega^{\mu-1 / 2}} \exp \left(-\frac{\mu r^{\alpha}}{\Omega}\right), \\
T_{R}(r) & \approx \frac{\Gamma\left(\mu, \mu r^{\alpha} / \Omega\right) \Omega^{\mu-1 / 2}}{\sqrt{2 \pi} f_{m} r^{\alpha(\mu-1 / 2)} \mu^{\mu-1 / 2}} \exp \left(\frac{\mu r^{\alpha}}{\Omega}\right)
\end{aligned}
$$

onde os parâmetros das fórmulas aproximadas, designados por $\alpha, \mu$, and $\Omega$ tem que ser obtidos para a aplicação apropriada. Perceba que (28) e (29) têm a mesma forma funcional de (7) e (8), respectivamente. Dessa forma, com o objetivo de tornar (28) e (29) boas aproximações, serão usados estimadores baseados em momentos para o cálculo de $\alpha, \mu$ e $\Omega$, como segue.

\section{A. Combinação por Ganho Igual}

Assuma, inicialmente, o conhecimento de $E(R), E\left(R^{2}\right)$ e $E\left(R^{4}\right)$. Então, estimadores baseados em momentos para os parâmetros $\alpha, \mu$ e $\Omega$ podem ser escritos baseados em [1], [2] como

$$
\begin{gathered}
\frac{\Gamma^{2}(\mu+1 / \alpha)}{\Gamma(\mu) \Gamma(\mu+2 / \alpha)-\Gamma^{2}(\mu+1 / \alpha)}=\frac{E^{2}(R)}{E\left(R^{2}\right)-E^{2}(R)}, \\
\frac{\Gamma^{2}(\mu+2 / \alpha)}{\Gamma(\mu) \Gamma(\mu+4 / \alpha)-\Gamma^{2}(\mu+2 / \alpha)}=\frac{E^{2}\left(R^{2}\right)}{E\left(R^{4}\right)-E^{2}\left(R^{2}\right)}, \\
\Omega=\left[\frac{\mu^{1 / \alpha} \Gamma(\mu) E(R)}{\sqrt{M} \Gamma(\mu+1 / \alpha)}\right]^{\alpha} .
\end{gathered}
$$

O sistema de equações transcendentais (30) e (31) têm que ser resolvidos numericamente para $\alpha$ e $\mu$. A maioria dos softwares de computadores possuem suas próprias rotinas que realizam esta tarefa de forma rápida e eficiente. Em MATHEMATICA, por exemplo, a função FindRoot pode ser usada para isso. Tendo obtido os parâmetros $\alpha$ e $\mu, \Omega$ é estimado através de (32).

Contudo, ainda temos que encontrar os momentos exatos $E(R), E\left(R^{2}\right)$ e $E\left(R^{4}\right)$ requeridos em (30), (31) e (32), que foram assumidos conhecidos. Para isso, usando expansão multinomial, estes momentos são obtidos a partir de (14) em termos de cada um dos momentos individuais $\alpha-\mu$ como [11]

$$
\begin{array}{r}
E\left(R^{n}\right)=\sum_{n_{1}=0}^{n} \sum_{n_{2}=0}^{n_{1}} \ldots \sum_{n_{M-1}=0}^{n_{M-2}}\left(\begin{array}{c}
n \\
n_{1}
\end{array}\right)\left(\begin{array}{l}
n_{1} \\
n_{2}
\end{array}\right) \ldots\left(\begin{array}{c}
n_{M-2} \\
n_{M-1}
\end{array}\right) \\
E\left(R_{1}^{n-n_{1}}\right) E\left(R_{2}^{n_{1}-n_{2}}\right) \ldots E\left(R_{M}^{n_{M-1}}\right), \quad
\end{array}
$$

onde os momentos $\alpha-\mu$ requeridos são dados em (4).

\section{B. Combinação por Razão Máxima}

Assim como para o caso EGC, nós propomos aproximar a LCR e AFD de $R$ pela LCR e AFD da envoltória $\alpha-\mu$ dado em (28) e (29), respectivamente. Perceba que como a técnica MRC consiste da soma de envoltórias ao quadrado, os estimadores são modificados tais que as condições de contorno sejam satisfeitas. Então, de [1], [2], chega-se a

$$
\begin{gathered}
\frac{\Gamma^{2}(\mu+2 / \alpha)}{\Gamma(\mu) \Gamma(\mu+4 / \alpha)-\Gamma^{2}(\mu+2 / \alpha)}=\frac{E^{2}\left(R^{2}\right)}{E\left(R^{4}\right)-E^{2}\left(R^{2}\right)}, \\
\frac{\Gamma^{2}(\mu+4 / \alpha)}{\Gamma(\mu) \Gamma(\mu+8 / \alpha)-\Gamma^{2}(\mu+4 / \alpha)}=\frac{E^{2}\left(R^{4}\right)}{E\left(R^{8}\right)-E^{2}\left(R^{4}\right)}, \\
\Omega=\left[\frac{\mu^{2 / \alpha} \Gamma(\mu) E\left(R^{2}\right)}{\Gamma(\mu+2 / \alpha)}\right]^{\alpha / 2} .
\end{gathered}
$$

Novamente, por expansão multinomial, os momentos exatos $E\left(R^{2}\right), E\left(R^{4}\right)$ e $E\left(R^{8}\right)$ requeridos em (34), (35) e (36) podem ser encontrados a partir de (22) como

$$
\begin{array}{r}
E\left(R^{2 n}\right)=\sum_{n_{1}=0}^{n} \sum_{n_{2}=0}^{n_{1}} \ldots \sum_{n_{M-1}=0}^{n_{M-2}}\left(\begin{array}{c}
n \\
n_{1}
\end{array}\right)\left(\begin{array}{l}
n_{1} \\
n_{2}
\end{array}\right) \ldots\left(\begin{array}{c}
n_{M-2} \\
n_{M-1}
\end{array}\right) \\
E\left(R_{1}^{2\left(n-n_{1}\right)}\right) E\left(R_{2}^{2\left(n_{1}-n_{2}\right)}\right) \ldots E\left(R_{M}^{2\left(n_{M-1}\right)}\right) .
\end{array}
$$




\section{Resultados NumÉricos}

Nesta seção, alguns gráficos ilustram as expressões aqui obtidas. A validade das mesmas é feita comparando as curvas teóricas com os resultados de simulação. Como será observado, há uma excelente concordância entre os resultados teóricos e os simulados. Além disto, outros resultados numéricos são plotados com o intuito de mostrar o bom ajuste das aproximações propostas.

Com o intuito de comparar as formulações exata e aproximada, Fig. 1, para o caso EGC, e Fig. 2, para o caso MRC, traçam a LCR normalizada (eixo da esquerda), $N_{R}(r) / f_{m}$, e AFD (eixo da direira), $T_{R}(r) f_{m}$, como função da envoltória. As curvas foram plotadas para $M=4, \alpha_{i}=1.5$ (caso EGC) e $\alpha_{i}=2.5$ (caso MRC), variando o parâmetro $\mu_{i}$. Note como as aproximações propostas produzem resultados muito bons, sendo verificado pela diferença mínima entre as curvas exatas e aproximadas. De fato, ambas as curvas são coincidentes para $\alpha_{i}=1$ (caso EGC) e $\alpha_{i}=2$ (caso MRC), independente do valor assumido por $\mu_{i}$. Ressalta-se que, quando $\alpha_{i}$ aumenta (de $1 \mathrm{a} \infty \rightarrow$ EGC, ou de 2 a $\infty \rightarrow$ MRC) ou $\alpha_{i}$ diminui (de 1 a $0 \rightarrow$ EGC, ou de 2 a $0 \rightarrow$ MRC), a diferença entre as curvas exatas e aproximadas aumenta, mas de uma maneira suave, de forma que um bom ajuste ainda é garantido. Tal comportamento não é mostrado nas figuras devido as mesmas serem traçadas para um único valor de $\alpha_{i}\left(\alpha_{i}=1.5 \rightarrow\right.$ EGC, $\alpha_{i}=2.5 \rightarrow$ $\mathrm{MRC})$. Além disso, para altos valores do parâmetro $\mu_{i}, \alpha_{i}$ pode ter um intervalo de variação ainda maior tal que uma ótima aproximação é ainda mantida.

As Figuras 3 e 4 traçam a LCR normalizada (eixo da esquerda) e AFD (eixo da direita), como uma função da envoltória, para as técnicas PSC, EGC e MRC. Os ramos são assumidos balanceados $\left(\Omega_{i}=\Omega=1\right)$, tendo parâmetros de desvanecimento $\alpha-\mu$ arbitrários. As curvas sem diversidade foram omitidas, porém, como já é bem conhecido na literatura, o uso de diversidade reduz drasticamente o efeito prejudicial do desvanecimento. Perceba que as técnicas EGC e MRC possuem desempenho similares, diferentemente da técnica PSC, que apresenta o pior desempenho. Tal degradação de desempenho relativo a técnica PSC torna-se ainda maior quando $M$ aumenta. Note ainda que para o caso no qual $M=2, \mu_{i}=2$ e $\alpha_{i}=2$ (Nakagami- $m$ ), a métrica AFD é praticamente coincidente para as três técnicas analisadas. As diferenças entre estas torna-se mais visível quando o parâmetro de não-linearidade $\alpha$ parte do caso linear $(\alpha=2)$.

Para $\mu_{i}=\mu=2$, Fig. 5 plota as curvas simuladas da LCR normalizada, como função da envoltória, para as técnicas PSC, EGC e MRC, usando dois ramos balanceados. Perceba a excelente concordância entre as curvas teóricas e simuladas. Para outras condições de desvanecimento, inúmeras simulações foram realizadas e, em todos os casos, um ótimo ajuste foi obtido.

\section{CONCLUSÕES}

Neste artigo, expressões exatas e aproximadas para a LCR e AFD das técnicas EGC e MRC sujeitas a desvanecimento $\alpha-\mu$ e usando $M$ ramos independentes, desbalanceados e não-idênticos foram obtidas. As aproximações propostas

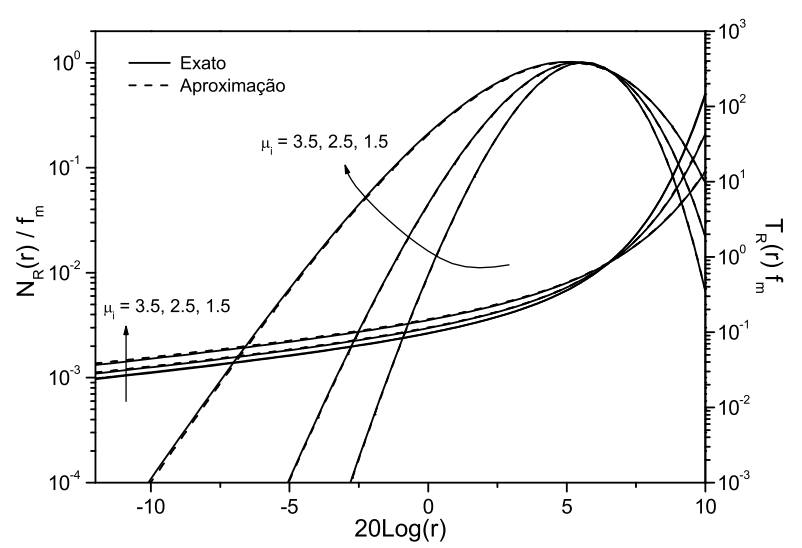

Fig. 1. LCR e AFD da técnica EGC para canais de desvanecimento $\alpha-\mu$ balanceados $\left(M=4, \alpha_{i}=1.5\right.$ e variando $\left.\mu_{i}=\mu\right)$.

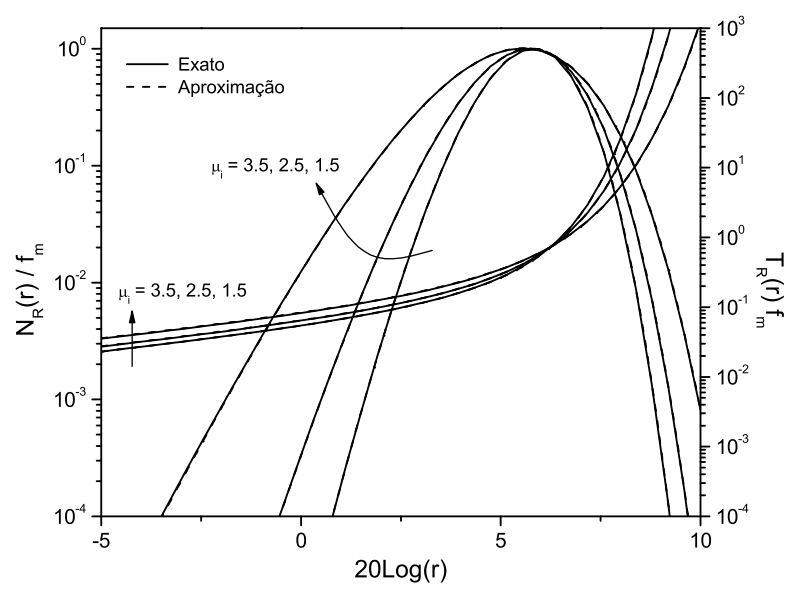

Fig. 2. LCR e AFD da técnica MRC para canais de desvanecimento $\alpha-\mu$ balanceados $\left(M=4, \alpha_{i}=2.5\right.$ e variando $\left.\mu_{i}=\mu\right)$.

mostraram-se ser altamente precisas. Além disso, expressões simples e em forma fechada para tais métricas (LCR e AFD) foram apresentadas para a técnica PSC no qual os canais estavam sujeitos as mesmas condições de desvanecimento das técnicas EGC e MRC.

As expressões exatas foram validadas reduzindo-as para alguns casos particulares, no qual as soluções são conhecidas, e por meio de simulação. Nossos resultados encontram aplicabilidade na análise e projeto de sistemas sem fio com diversidade operando em condições generalizadas de desvanecimento, onde ambos os fenômenos de não-linearidade e clusterização ocorrem.

\section{REFERÊNCIAS}

[1] M. D. Yacoub, The $\alpha-\mu$ distribution: A general fading distribution. IEEE Inter. Symp. on Personal, Indoor and Mobile Radio Commun., v. 2, p. 629-633, Sep., 2002. 


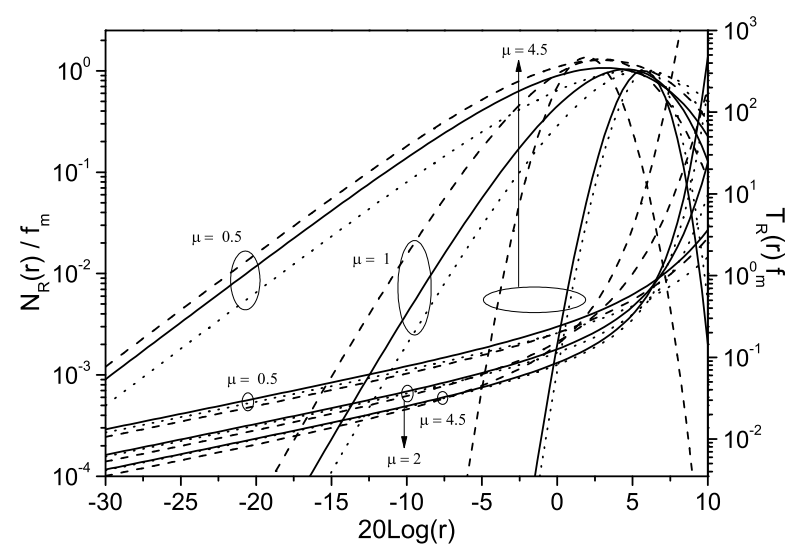

Fig. 3. LCR e AFD das técnicas PSC, EGC e MRC para canais de desvanecimento $\alpha-\mu$ balanceados (linhas sólidas $\rightarrow$ EGC, linhas pontilhadas $\rightarrow$ MRC, linhas tracejadas $\rightarrow$ PSC, $M=4, \Omega_{i}=1, \alpha_{i}=1.5$ e variando $\left.\mu_{i}=\mu\right)$.

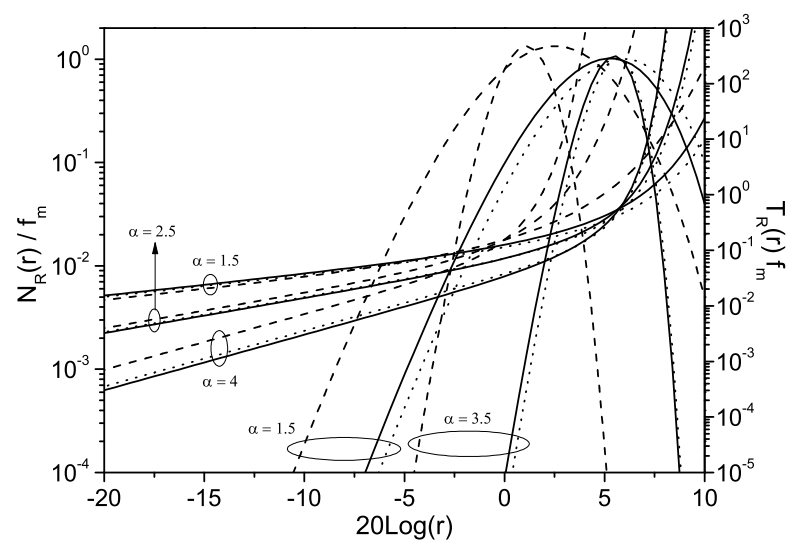

Fig. 4. LCR e AFD das técnicas PSC, EGC e MRC para canais de desvanecimento $\alpha-\mu$ balanceados (linhas sólidas $\rightarrow$ EGC, linhas pontilhadas $\rightarrow$ MRC, linhas tracejadas $\rightarrow$ PSC, $M=4, \Omega_{i}=1, \mu_{i}=2$ e variando $\left.\alpha_{i}=\alpha\right)$.

[2] M. D. Yacoub, The $\alpha-\mu$ distribution: a physical fading model for the Stacy distribution, IEEE Trans. Veh. Technol, v. 56, $\mathrm{n}^{\circ}$ 1, p. 27-34, Jan., 2007.

[3] G. Fraidenraich, J. C. S. Santos Filho, and M. D. Yacoub, Second-order statistics of maximal-ratio and equal-gain combining in Hoyt fading, IEEE Commun. Lett., v. 9, $\mathrm{n}^{\circ}$ 1, p. 19-21, Jan., 2005.

[4] G. Fraidenraich, M. D. Yacoub, and J. C. S. Santos Filho, Second-order statistics of maximal-ratio and equal-gain combining in Weibull fading, IEEE Commun. Lett., v. 9, n 6, p. 499-501, Jun., 2005.

[5] M. D. Yacoub, C. R. C. M. da Silva, and J. E. V. Bautista, Secondorder statistics for diversity-combining techniques in Nakagami-fading channels, IEEE Trans. Veh. Technol., v. 50, n 6, p. 1464-1470, Nov., 2001.

[6] S. O. Rice, Mathematical analysis of random noise, Bell System Technical Journal, v. 23, p. 282-332, Jul., 1944.

[7] W. C. Jakes, Microwave Mobile Communications, 1974

[8] L. Yang and M. -S. Alouni, Level crossing rate over multiple independent random processes - an extension of the applicability of the Rice formula. Proc. IEEE Global Telecommun. Conf., San Francisco, USA, Dez., 2005.

[9] A. Papoulis, Probability, Random Variables, and Stochastic Processes,

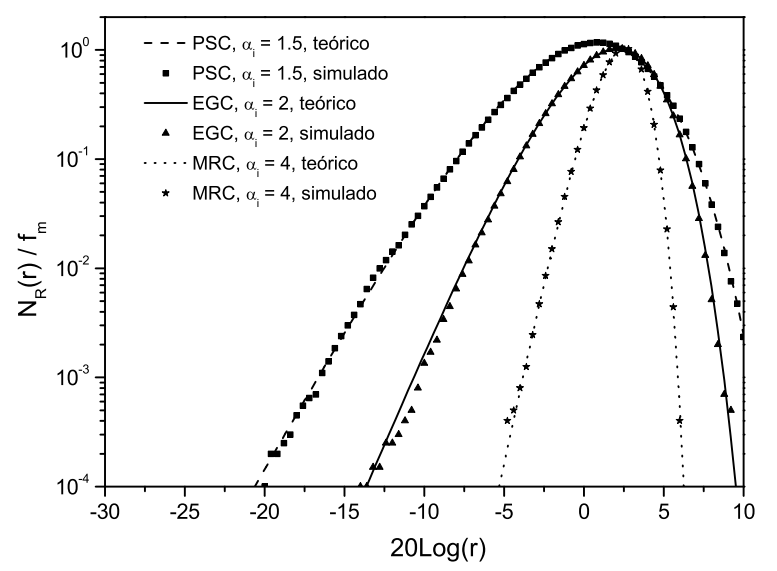

Fig. 5. Comparação entre as curvas teóricas e simuladas para PSC, EGC, e MRC com $M=2$ e usando $\mu=2$.

4th edition, 1974

[10] D. G. Brennan, Linear diversity combining techniques, v. 47, p. 10751102, Jun., 1959.

[11] J. C. S. Santos Filho and M. D. Yacoub, Simple precise approximations to Weibull sums, IEEE Commun. Lett., v. 10, $\mathrm{n}^{\circ}$ 8, p. 614-616, Aug., 2006.

[12] C.-D. Iskander and P. T. Mathiopoulus, Analytical level crossing rates and average fade durations for diversity techniques in Nakagami fading channels, IEEE Trans. Commun., v. 50, n 8, p. 1301-1309, Aug., 2002.

\section{APÊNDICE I}

O objetivo deste Apêndice é encontrar a variância da PDF condicional de $\dot{R}_{i}$ dado $R_{i}$. A partir de (1), temos que

$$
R_{i}^{\alpha_{i}}=\sum_{l=1}^{n_{i}}\left(X_{i l}^{2}+Y_{i l}^{2}\right) .
$$

Derivando cada lado da igualdade em (38), temos

$$
\alpha_{i} R_{i}^{\alpha_{i}-1} \dot{R}_{i}=\sum_{l=1}^{n_{i}}\left(2 X_{i l} \dot{X}_{i l}+2 Y_{i l} \dot{Y}_{i l}\right) .
$$

Rearranjando os termos e aplicando o operador variância condicional,

$$
\sigma_{\dot{R}_{i}}^{2}=4 \sum_{l=1}^{n_{i}} \frac{X_{i l}^{2}+Y_{i l}^{2}}{\alpha_{i}^{2} R_{i}^{2 \alpha_{i}-2}} \dot{\sigma}_{i}^{2} .
$$

Sabendo que ${\dot{\sigma_{i}}}^{2}=2 \pi^{2} f_{m}^{2} \sigma_{i}^{2}=\pi^{2} f_{m}^{2} \Omega_{i} / \mu_{i}$, obtém-se

$$
\sigma_{\dot{R}_{i}}^{2}=4 \pi^{2} f_{m}^{2} \sum_{l=1}^{n_{i}} \frac{X_{i l}^{2}+Y_{i l}^{2}}{\alpha_{i}^{2} \mu_{i} R_{i}^{2 \alpha_{i}-2}} \Omega_{i} .
$$

Substituindo (38) em (41), a demonstração está concluída. Portanto, a variância da PDF condicional de $\dot{R}_{i}$ dado $R_{i}$ é expressa por

$$
\sigma_{\dot{R}_{i}}^{2}=\frac{R_{i}^{2-\alpha_{i}}}{\alpha_{i}^{2} \mu_{i}} 4 \pi^{2} f_{m}^{2} \Omega_{i}
$$

